\title{
Contaminantes microbiológicos en un mercado del sur de Montería: Un riesgo para la salud pública
}

\section{Microbial contamination in a market in southern Montería: A risk to public health}

Fecha de recepción: 12 de diciembre de 2016 Fecha de aprobación: 16 de mayo de 2017
Camilo A. Guzmán T. ${ }^{1}$

Virginia Consuelo Rodríguez-Rodríguez ${ }^{2}$ Alfonso Calderón-Rangel ${ }^{3}$

DOI: http://doi.org/10.19053/01228420.v14.n2.2017.7161

\section{Resumen}

Las enfermedades transmitidas por alimentos (ETA) son alteraciones agudas causadas por microorganismos patógenos o sustancias tóxicas provenientes de alimentos contaminados; pueden producir diarrea masiva, vómitos, deshidratación e incluso la muerte. Las ETA son un problema muy frecuente y de mayor impacto a nivel global. Una epidemia por ETA perjudica el comercio y el turismo, dado que influye negativamente en la confianza de los consumidores, provocando pérdidas económicas y problemas legales. Dentro de los patógenos involucrados en ETA encontramos al Staphylococcus aureus, productor de enterotoxinas, y las enterobacterias; todos estos patógenos producen diarrea, vómito, deshidratación $y$, en casos extremos, la muerte, si no se recibe tratamiento médico oportuno. Mediante un estudio analítico transversal, se tomaron muestras de mesones, de manos de los manipuladores y de hortalizas. Igualmente, se recolectaron muestras de agua residual por duplicado en los drenajes a la salida de los pabellones de carne y de hortalizas, para determinar Vibrio cholerae. Todas las muestras se transportaron inmediatamente en refrigeración al laboratorio para diagnóstico microbiológico convencional. En todas las muestras de las manos de los manipuladores de alimentos y de las superficies de los mesones se aisló Staphylococcus spp.; igualmente, se aislaron enterobacterias fermentadoras y no fermentadoras. Las enterobacterias aisladas de las manos de los manipuladores fueron: Citrobacter spp. $(31,25 \%)$, Proteus vulgaris $(18,75 \%)$, Klebsiella $(6,25 \%)$ y Enterobacter spp. $(6,25 \%)$. No se aisló $V$. cholerae, pero se encontraron quistes de Entamoeba histolytica. El aislamiento de $S$. aureus y de enterobacterias, de las

1 M. Sc. Universidad de Córdoba (Montería, Colombia). cguzman@unicordoa.edu.co. ORCID: 0000-0002-0295-4647. 2 M. Sc. Universidad de Córdoba (Montería, Colombia).vrodriguez@correo.unicordoba.edu.co. ORCID: 0000-00020295-4647.

3 M. Sc. Universidad de Córdoba (Montería, Colombia). acalderonr@correo.unicordoba.edu.co. ORCID: 0000-00016503-8323. 
manos de los manipuladores de alimentos y de las superficies de los mesones, así como de E. histolytica, de las verduras, evidencia la existencia de un riesgo para la salud pública en el Mercado del Sur de la ciudad de Montería (Córdoba), donde se realizó el estudio.

Palabras clave: alimentos; enterobacteriaceae; ETA; parásitos; patógenos; verduras.

\begin{abstract}
Foodborne diseases (FBD) are acute alterations caused by pathogenic microorganisms or toxic substances found in contaminated food that may produce massive diarrhea, vomiting, dehydrating, and even death. The FBD are a frequent health problem and have a large global impact. A FBD epidemic harms trade and tourism, due to its negative impact on the consumers' confidence, which causes economic losses and legal problems. Staphylococcus aureus, enterotoxins producer, enterobacteria, and protozoans are some of the pathogens involved in FBD; all of them cause diarrhea, vomiting, dehydration and, in extreme cases, death if treatment is not received timely. We conducted a transverse analytical study, for which, we sample mesons, the hands of food handlers, and vegetables such as lettuce and cabbage. Likewise, we collected samples (in duplicate) from wastewater streams in the drain outlets near to meat and vegetables pavilions to determine the presence of Vibrio cholera. All samples were immediately refrigerated and transported to the laboratory for conventional microbiological diagnosis. We isolated Staphylococcus spp. and fermenter and non-fermenter enterobacteria from the hands of food handlers and mesons surfaces. The enterobacteria isolated from the hands of the handlers were Citrobacter spp. $(31,25$ $\%)$, Proteus vulgaris (18,75\%), Klebsiella (6,25\%) and Enterobacter spp. (6,25\%). V. cholerae was not isolated, but Entamoeba histolytica cysts were found. Finding $S$. aureus and enterobacteria in the hands of food handlers and mesons surfaces, and E. histolytica in the vegetables constitutes a risk to public health.
\end{abstract}

Keywords: enterobacteriaceae; FBD; food; noxae; parasites; vegetables.

\title{
Como citar:
}

Guzmán-T. CA., Rodríguez-Rodríguez VC., Calderón-Rangel A. Contaminantes microbiológicos en un mercado del sur de Montería: Un riesgo para la salud pública. Rev. Cien. Agri. 2017; 14(2): 89-97. 


\section{Introducción}

Las enfermedades transmitidas por alimentos (ETA) se definen como el síndrome producido por el consumo de alimentos o bebidas contaminados que alteran la salud del consumidor, individualmente o colectivamente; son agudas, por la ingestión de alimentos contaminados que actúan como vehículos de transmisión de patógenos o sustancias tóxicas que se incorporan de modo accidental, incidental o intencional en cualquier momento desde su fabricación hasta el consumo $(1,2)$, y en su gran mayoría son infecciones ocasionadas por virus, bacterias y parásitos que provienen de los alimentos; otras son los envenenamientos producidos por las toxinas o productos químicos nocivos que han contaminado los alimentos (2).

Las ETA constituyen una problemática sanitara muy frecuente y de mayor impacto a nivel global; afligen, principalmente, a infantes, mujeres embarazadas, ancianos y población en general de estratos pobres. Una epidemia por ETA perjudica el comercio y el turismo, pues lesiona la confianza de los consumidores, provocando pérdidas económicas y problemas legales $(2,3)$.

Los microorganismos responsables de brotes de ETA son numerosos; entre ellos se cuentan virus entéricos, como adenovirus, rotavirus, norovirus y hepatitis, y bacterias, como Salmonella spp., S. typhi y S. paratyphi, V. cholerae, E. coli enteropatógena, Clostridium spp, L. monocytogenes, $Y$. enterocolítica, $B$. cereus y Shigella spp., entre otras, que producen enfermedades de interés en salud pública como fiebre tifoidea y paratifoidea, cólera, hepatitis A, salmonelosis, shigelosis, yersiniosis y listeriosis, entre otras $(2,4)$.

Los riesgos de ETA se han incrementado debido a la centralización de la producción de alimentos, al aumento del consumo colectivo de alimentos y a la expansión del comercio internacional y del turismo. Muchos brotes de ETA no se reportan, y si se han reportado no se llega a identificar al agente causal, por las deficiencias del sistema de vigilancia y la falta de métodos de diagnóstico apropiados $(2,5)$. Se calcula que cada año en el mundo se enferman por ETA 600 millones de personas, casi uno de cada diez habitantes del planeta, y mueren por esta causa 420000; cuando se ajustan estas cifras en función de la discapacidad, se encuentra que se pierden 33 millones de años de vida (6).

Dentro de los factores de riesgo que pueden desencadenar un brote de ETA se encuentra la manipulación incorrecta o negligente de quienes se desempeñan como preparadores de alimentos. La causa primaria de contaminación es la falta de higiene de los manipuladores y de los utensilios o superficies donde se elaboran las preparaciones (7); otros factores de riesgo identificados son la conservación y el almacenamiento inadecuados, como las fallas en la cadena de frío (8).

En las frutas y hortalizas, la contaminación microbiana se origina por el contacto con aguas, suelos, equipos, animales o personas que favorecen la vehiculización de los contaminantes durante el cultivo, la cosecha, el transporte, el almacenamiento y la venta; por ello es necesario un seguimiento del producto en todo momento (9). Entre los alimentos que se ingieren crudos está la lechuga (Lactuca sativa), hortaliza en la que se ha determinado el mayor porcentaje de formas parasitarias $(10,11)$, por lo cual, si no se le hace una adecuada higienización previa (12), representa un riesgo para la transmisión de enteropatógenos $(11,13,14)$.

La presencia de los patógenos en vegetales se puede prevenir con el lavado y escurrido, cuyo objetivo es eliminar la contaminación por insectos, suciedad y partículas de tierra, así como los residuos de plaguicidas, fertilizantes y otros productos fitoquímicos, con el fin de reducir la carga microbiana y mejorar el aspecto del producto (9).

EI S. aureus es uno de los microorganismos que causa variedad de enfermedades en seres humanos y animales (15); se encuentra en los alimentos por contaminación durante su obtención, almacenamiento o comercialización, al ser manipulados por personas contaminadas $(16,17$, 18, 19). Dentro de los mecanismos de virulencia encontramos las enterotoxinas estafilocócicas (SE); se han descrito más de $15 \mathrm{SE}$, de A (SEA) a J (SEJ) 
$(20,21)$. Ciertas SE son termoestables después de la pasteurización (22). Las que se vinculan más frecuentemente a intoxicaciones alimentarias son SEA, SEB, SEC1, SEC2, SEC3, SED y SEE $(23,24)$.

La familia de las Enterobacteriaceas está conformada por Escherichia, Enterobacter, Klebsiella, Serratia, Edwarsiella, Citrobacter, Salmonella y otros géneros, que viven como saprófitos independientes o como bacterias intestinales. Estas bacterias pueden causar al humano infecciones oportunistas, infecciones de piel y tejidos blandos, enfermedad diarreica aguda y otras enfermedades severas (25); además, pueden sobrevivir en los alimentos y conservan su patogenicidad elevada, especialmente Salmonella (26). La presencia de esta familia en alimentos representa un problema de salud pública, e indica que estos han sido elaborados en condiciones higiénicas deficientes, manipulados de manera incorrecta y mantenidos a temperatura ambiente durante periodos prolongados (27).

El género Vibrio comprende varias especies de bacilos gramnegativos de importancia médica; de todas ellas merece especial atención $V$. cholerae, que es un bacilo gramnegativo anaerobio facultativo responsable de diarrea masiva, vómito y deshidratación, que puede ser grave e incluso llevar a la muerte si no se recibe tratamiento médico oportuno $(28,29)$.

La capacitación de los productores y de su personal de apoyo sobre prácticas preventivas y correctivas puede disminuir los riesgos de contaminación microbiológica durante todos los procesos productivos; es el caso de los conocimientos básicos sobre las Buenas Prácticas Agrícolas (BPA), como, por ejemplo, el riego con agua de calidad e impedir el contacto con heces durante la producción, cosecha y postcosecha (30). En Colombia, el Sistema Nacional de Vigilancia en Salud Pública (Sivigila) empezó en el año 2000 el monitoreo de brotes de ETA, con la notificación de 2983 casos, y en el año 2001 registró 5381 casos; en la Figura 1 se observa que hay una tendencia creciente en el número de casos (2).
El objetivo de este trabajo fue detectar $S$. aureus y enterobacterias en manos de los manipuladores de alimentos y en las superficies de los mesones de los pabellones de carne y hortalizas, y en algunas hortalizas en un mercado del sur de Montería.

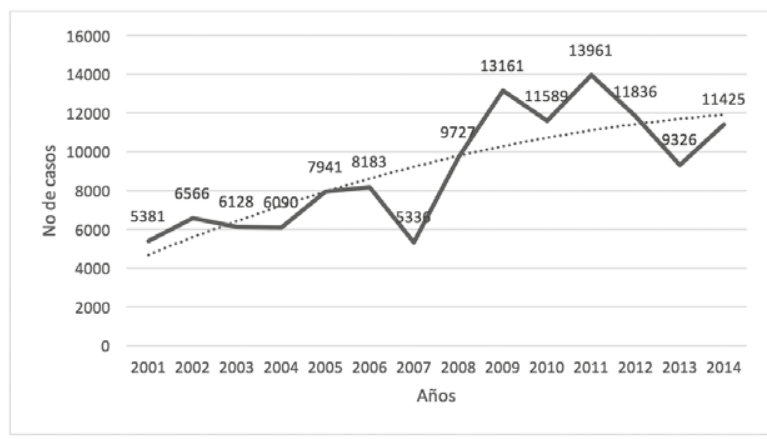

Fig. 1. Notificación de brotes de ETA en Colombia entre 2001 y 2014.

\section{Materiales y métodos}

Mediante un estudio analítico transversal y un diseño de conveniencia, se tomaron 24 muestras de las manos de los manipuladores de alimentos y de las superficies de los mesones de los pabellones de carnes y hortalizas; igualmente, se tomaron ocho muestras de hortalizas (lechugas y repollo) y cuatro de agua residual de estos pabellones. Todas las muestras se tomaron en el Mercado del Sur de Montería.

La toma de las muestras de las manos de los manipuladores y de las superficies de los mesones se hizo por medio de hisopados estériles, que se frotaron sobre estas superficies, se rotularon y, posteriormente, se depositaron en tubos estériles de ensayos que contenían $1 \mathrm{ml}$ de solución salina estéril. La toma del agua residual, para determinar $V$. cholerae, se hizo mediante el empleo de mechas de gasa estériles, las cuales se colocaron por duplicado a la salida de los drenajes de los pabellones de carne y de hortalizas y se retiraron 48 y 72 horas después; estas gasas se depositaron en frascos estériles tapa azul con $300 \mathrm{ml}$ de agua peptonada alcalina $(\mathrm{pH}=8,7)$. Para recolectar las muestras de las hortalizas (lechugas y repollo), estas se lavaron individualmente con una solución 
salina estéril que se depositó en un tubo de ensayo tapa rosca estéril. Todas las muestras se transportaron en refrigeración al laboratorio.

\section{A. Identificación microbiológica}

Los hisopados de las muestras de las manos de los operarios y de las superficies se sembraron por agotamiento en placas de agar sangre, agar manitol salado y agar Mac Conkey, y se incubaron a 37 ${ }^{\circ} \mathrm{C}$ durante 24 horas. Se realizó la caracterización macroscópica de las características de las colonias, la cual se complementó con la coloración de Gram y algunas pruebas bioquímicas básicas. A los cocos Gram positivos, por la coloración de Gram y por la presencia de colonias amarillas o blancas, se les implementó la catalasa y la coagulasa, y se reportaron como Staphylococcus coagulasa positivos (SCP), cuando fueron catalasa y coagulasa positivos, y Staphylococcus coagulasa negativos ( $\mathrm{SCN}$ ), cuando fueron catalasa positivos y coagulasa negativos. Los bacilos que crecieron en el agar Mac Conkey se clasificaron como fermentadores o lactosa positivos (colonias rosadas) y no fermentadores o lactosa negativos (colonias incoloras); a las colonias lactosa negativas se les implementó las pruebas bioquímicas agar TSI, agar LIA, agar citrato de Simmons, agar urea, agar SIM y caldo MR-VP $(31,32)$.

Los frascos con las gasas con agua peptonada alcalina de los desagües se llevaron a incubación por 24 horas a $37^{\circ} \mathrm{C}$; luego, se tomó una alícuota con un asa bacteriológico y se sembró por agotamiento en agar Mac Conkey y agar TCBS, que es selectivo para las especies de Vibrio. Para confirmar el resultado, se realizó la prueba de la oxidasa y la prueba de la cuerda o filamentosidad; según esta, al agregar el desoxicolato de sodio al $0.5 \%$ se formará una cuerda mucoide que debe sostenerse durante cinco segundos (33).

El líquido obtenido del lavado de las verduras (lechuga y repollo) se centrifugó a 3.500 revoluciones durante 5 minutos, para lograr la concentración de los diferentes elementos (huevos, larvas y quistes), y se descartó el sobrenadante; con el sedimento se hizo una extensión con suero fisiológico en una lámina portaobjetos, y para lograr una mejor visualización se adicionó lugol y se observó con el objetivo de 40X (34).

\section{Resultados y discusión}

En la totalidad de las muestras de las manos de los manipuladores de alimentos, sembradas en agar sangre, se aislaron Staphylococcus; el $50 \%$ de estos fueron coagulasa positivos (SCP), y el otro $50 \%$, coagulasa negativos ( $\mathrm{SCN}$ ). En todas las muestras de las superficies de los mesones se aislaron Staphylococcus; el $44 \%$ de estos aislamientos fueron SCP, y el $66 \%$ SCN.

El aislamiento de SCP de las manos de los manipuladores de alimentos y de las superficies de los mesones indica un riesgo para la salud pública, por la producción de enterotoxinas, de las cuales se han descrito más de quince $(20,21)$. Estas enterotoxinas pueden causar enfermedades transmitidas por alimentos como enterotóxicosis y enfermedad diarreica aguda $(23,24)$. La presencia de $S$. aureus en los alimentos se debe al uso de materia prima contaminada, como, por ejemplo, de leche proveniente de vacas con mastitis o de otros productos contaminados por los manipuladores, por prácticas inadecuadas de manufactura $(35,36)$ o una excesiva e inadecuada manipulación (37).

Los manipuladores de alimentos son una fuente muy importante de contaminación de $S$. aureus $(19,38)$; se ha aislado $S$. aureus de la piel y las mucosas de humanos y animales; igualmente, está presente en fosas nasales, garganta, cabello y piel de las personas sanas $(39,40,41)$, aunque en este estudio no se pudo comprobar este tipo de contaminación.

Inicialmente, el $75 \%$ de los bacilos Gram negativos se clasificaron como no fermentadores, y el $25 \%$, como fermentadores; al implementar las pruebas bioquímicas, el 62,5\% fueron colonias verdaderamente no fermentadoras, y el $37,5 \%$, colonias fermentadoras (Tabla I). La presencia de enterobacterias es indicador de contaminación fecal, y su ausencia es indicador de Buenas Prácticas de Manufactura (BPM); recuentos elevados indican una elaboración deficiente o una contaminación posterior, o ambas; el hallazgo 
de Shigella spp. en determinadas muestras se debió a la contaminación de los manipuladores de alimentos. Igualmente, se aislaron otras enterobacterias, como Citrobacter freundii, Pantotea agglomerans y E. coli (42), patógenos que representan un riesgo higiénico-sanitario (43). Las enterobacterias aisladas en el presente estudio corresponden a microorganismos oportunistas, es decir, no son bacterias absolutamente patógenas, ya que su potencial de virulencia es bajo en comparación con otras especies de los géneros aislados. En el Caribe colombiano se determinó que la alta contaminación de los productos con enterobacterias es consecuencia de las malas condiciones sanitarias de su manufactura (44).

Tabla I. Distribución de enterobacterias fermentadoras y no fermentadoras en las manos de manipuladores.

\begin{tabular}{|l|l|c|c|}
\hline Muestra & Enterobacteria & CVNF & CVF \\
\hline 3 & Citrobacter spp. & $\checkmark$ & \\
\hline 4 & Klebsiella spp. & & $\checkmark$ \\
\hline 7 & Citrobacter spp. & $\checkmark$ & \\
\hline 9 & Citrobacter spp. & $\checkmark$ & \\
\hline 10 & Citrobacter spp. & $\checkmark$ & \\
\hline 11 & Proteus spp. & $\checkmark$ & \\
\hline 13 & Enterobacter spp. & & $\checkmark$ \\
\hline 14 & Proteus spp. & $\checkmark$ & \\
\hline 15 & Proteus spp. & $\checkmark$ & \\
\hline 16 & Citrobacter spp. & $\checkmark$ & \\
\hline
\end{tabular}

$\mathrm{CVNF}=$ Colonias verdaderamente no fermentadoras.

$\mathrm{CVF}=$ Colonias verdaderamente fermentadoras.

Las enterobacterias aisladas de las manos de los manipuladores fueron: Citrobacter spp. $(31,25$ $\%)$, P. vulgaris $(18,75 \%)$, Klebsiella spp. $(6,25$ $\%)$ y Enterobacter spp. (6,25\%). En la Tabla II se presenta la distribución de las enterobacterias fermentadoras y no fermentadoras de las manos de los manipuladores.
Tabla II. Distribución de las enterobacterias fermentadoras y no fermentadoras en superficie de mesones.

\begin{tabular}{|l|l|c|c|}
\hline Muestra & Enterobacteria & CVNF & CVF \\
\hline 2 & Proteus spp. & $\checkmark$ & \\
\hline 3 & Citrobacter spp. & $\checkmark$ & \\
\hline 5 & Citrobacter spp. & $\checkmark$ & \\
\hline 7 & Klebsiella spp. & & $\checkmark$ \\
\hline 8 & Proteus spp. & & \\
\hline 9 & Proteus spp. & & \\
\hline 10 & Citrobacter spp. & & \\
\hline 12 & Proteus spp. & & \\
\hline 13 & Klebsiella spp. & & $\checkmark$ \\
\hline 14 & Proteus spp. & $\checkmark$ & \\
\hline 15 & Proteus spp. & $\checkmark$ & \\
\hline 16 & Citrobacter spp. & $\bullet$ & \\
\hline CVNF $=$ & Colonias verdaderamente no \\
& \multicolumn{4}{|c|}{ fermentadoras. }
\end{tabular}

$\mathrm{CVF}=$ Colonias verdaderamente fermentadoras

De las muestras sembradas en las cajas Agar TCBS por duplicado, en las mechas de las cajas uno (48 horas) no se observó crecimiento de colonias sospechosas para Vibrio spp., mientras que en las mechas de las cajas dos (72 horas) se observó crecimiento de colonias sospechosas (color amarillo); a estas colonias sospechosas se les implementó la prueba de la cuerda, cuyo resultado fue negativo.

Se determinó que en el $12,5 \%(2 / 16)$ de las verduras estudiadas sólo se encontró la presencia de dos quistes en Entamoeba histolytica, y en el $81,25 \%$, protozoos ambientales sin importancia clínica en humanos; la ingestión de quistes maduros de E. histolytica, a partir del agua, de los alimentos o de las manos contaminadas con heces causa enfermedad (45). En frutas y hortalizas también se detectó presencia de E. histolytica, pero la mayor frecuencia se encontró en las hortalizas (12); sin embargo, se observó que $E$. histolytica fue la más abundante en las verduras. La detección de E. histolytica indica que en algún 
momento del proceso de obtención, manipulación, conservación, transporte o disposición final de estos vegetales existió contacto con materia fecal. Puede considerarse que la contaminación por E. histolytica está muy determinada por el nivel higiénico sanitario de la población y por su presencia en el ambiente (46). En Costa Rica, E. histolytica se determinó en el 3,8 \% de las muestras de lechuga (47). En Colombia, en manipuladores de alimentos se determinó que la mayor prevalencia fue $E$. histolytica, seguida por $B$. hominis, y que los casos de infestación múltiple fueron $E$. histolytica y $E$. coli (48), y en ventas ambulantes de alimentos (chorizo frito, ensaladas de frutas, yogurt con cereal, arepa rellena y pincho de carne) y en manipuladores de alimentos se detectaron parásitos como E. histolytica/E. dispar, que tienen importancia en salud pública (49).

V. cholerae se ha aislado de alimentos (50) y de varias fuentes de agua (51), cuyo consumo puede producir una epidemia (52). Las aguas residuales se han constituido en un punto importante para la emergencia de este patógeno (53), ya que tiene un doble ciclo de vida, intestinal y acuático (54). El no haber aislado $V$. cholerae de las fuentes de agua residuales evaluadas es un buen indicador ambiental, ya que nos dice que, a pesar de la alta contaminación de los pabellones de carne y de frutas en el mercado del sur de Montería, no hay presencia de este patógena. Sin embargo, es importante no descuidar este patógeno, por lo que es necesario mantener la vigilancia epidemiológica.

En alimentos, la contaminación puede presentarse en los diferentes procesos productivos, como la siembra, cosecha, almacenamiento, venta $y$ consumo, y producir ETA, que constituyen un problema importante de salud pública. En Estados Unidos, combinando los principales patógenos conocidos y los no específicos, el estimado general anual de la carga total de enfermedades causada por alimentos contaminados es de 47,8 millones de enfermedades, 127839 hospitalizaciones y 3037 muertes (4). En Colombia, el Sivigila ha reportado un aumento de casos de ETA durante los últimos 15 años (2), aunque en muchos casos no se ha establecido su etiología (Fig. 1). La violación de las buenas prácticas de manufactura, las prácticas no adecuadas y los hábitos higiénicos imprudentes en manipuladores de alimentos son elementos que influyen en la presentación de brotes de ETA (55).

\section{Conclusiones}

En todas las muestras de mesones y manos de los manipuladores de carnes se aisló de $S$. aureus, lo que evidencia un riesgo para la salud de los consumidores. Las colonias verdaderamente no fermentadoras (CVNF), correspondientes a microorganismos ampliamente distribuidos en la naturaleza, se pueden encontrar en agua, suelo y distintos tipos de superficie, constituyendo microorganismos ambientales. La existencia de quistes de E. histolytica en las verduras puede producir enfermedad diarreica aguda. No se detectó la presencia de $V$. cholerae de las fuentes de agua residuales evaluadas.

\section{Referencias}

(1) Borbolla M., Vidal M., Piña O., Ramírez I., Vidal J. Contaminación de los alimentos por Vibrio cholerae, coliformes fecales, Salmonella, hongos, levaduras y Staphylococcus aureus en Tabasco durante 2003. Salud Tab. 2004; 10(1-2): 221-232.

(2) Instituto Nacional de Salud (INS). Informe final del evento Enfermedades Transmitidas por Alimentos, Colombia, 2015. Dirección de Vigilancia y Análisis del Riesgo en Salud Pública. Informe evento FOR-R02.4000-001, 2015.

(3) Uribe MA., Ospino RJ., Forero NCP. Estudio de brote de enfermedad transmitida por alimentos. Rev. Cienc. cuidad. 2014; 11(1):7-15.

(4) Centro para el Control Enfermedades (CDC). Estimaciones sobre enfermedades transmitidas por alimentos en los EE. UU. en el 2011. Disponible en: http://www.cdc.gov/spanish/Datos/ EnfermedadesAlimentos/.

(5) Instituto Nacional de Salud (INS). Protocolo de vigilancia en salud pública enfermedades transmitidas por alimentos. Vigilancia y análisis del riesgo en salud pública protocolo de vigilancia en salud pública. Enfermedades Transmitidas por alimentos. PRO-R02.001 Versión 02. 2016.

(6) Organización Mundial de la Salud (OMS). Inocuidad de los alimentos. Nota descriptiva, N. ${ }^{\circ}$ 399. 2015. Disponible en: http://www.who.int/mediacentre/factsheets/fs399/es/.

(7) Lues JFR., Van Tonder I. The occurrence of indicator bacteria on hands and aprons of food handlers in the delicatessen sections of a retail group. Food Control. 2007; 18: 326-332. DOI: http://doi. org/10.1016/j.foodcont.2005.10.010.

(8) Guerrero MJA. Informe final del evento Enfermedades Transmitidas por Alimentos, Colombia, 2015. Dirección de Vigilancia y Análisis del Riesgo en Salud Pública. Instituto Nacional de Salud. 
(9) Cámara HM., Cortés SMM., Torija IE. Nutrición y Salud: Frutas y verduras fuentes de salud. Nueva Imprenta, S.A. Madrid, España, 2008.

(10) Traviezo-Valles L., Dávila J., Rodríguez R., Perdomo O., Pérez J. Contaminación enteroparasitaria de lechugas expendidas en mercados del estado Lara, Venezuela. Parasitol. Latinoam. 2004; 59(3): 167-170. DOI: http://doi.org/10.4067/S071777122004000300014.

(11) Barrantes K., Achí A. Calidad microbiológica y análisis de patógenos (Shigella y Salmonella) en lechuga. Rev. Soc. Ven. Microbiol. 2011; 31: 31-36.

(12) Camargo CNA., Campuzano S. Estudio piloto de detección de parásitos en frutas y hortalizas expendidas en los mercados públicos y privados de la ciudad de Bogotá D.C. Nova. 2006; 4(5): 111-116.

(13) Sivapalasingam S., Friedman CR., Cohen L., Tauxe RV. Fresh produce: a growing cause of outbreaks of food borne illness in the United States, 1973 through 1997. J. Food Prot. 2004; 67(10): 2342-2353. DOI: http://doi.org/10.4315/0362028X-67.10.2342.

(14) Beuchat LR. Ecological factors influencing survival and growth of human pathogens on raw fruits and vegetables. Microb Infect. 2002; 4: 413-23. DOI: http://doi.org/10.1016/S12864579(02)01555-1.

(15) Da Silva ER., Boechat JUD., Silva N. Coagulase gene typing of Staphylococcus aureus isolated from cows with mastitis in southeastern Brazil. Can. J. Vet. Res. 2005; 69(4): 260-264.

(16) Boynukara B., Gulhan T., Alisarli M., Gurturk K., Solmaz H. Classical enterotoxigenic characteristics of Staphylococcus aureus strains isolated from bovine subclinical mastitis in Van, Turkey. Int. J. Food Microbiol. 2008; 125(2): 209-211. DOI: http://doi.org/10.1016/j.ijfoodmicro.2008.03.024.

(17) Elmoslemany A., Keefe G., Dohoo I., Jayarao B. Risk factors for bacteriological quality of bulk tank milk in Prince Edward Island dairy herds. Part 2: Bacteria count specific risk factors. J. Dairy Sci. 2009; 92(6): 2644-2652. DOI: http://doi.org/10.3168/ jds.2008-1813.

(18) Rall VL., Sforcin JM., Augustini VCM., Watanabe MT., Fernandez Jr. A., Rall R., et al. Detection of enterotoxin genes of Staphylococcus sp. isolated from nasal cavities and hands of food handlers. Braz. J. Microbiol. 2010; 41: 59-65. DOI: http://doi. org/10.1590/S1517-83822010000100011.

(19) Jordá GB., Marucci R., Guida A., Pires PS., Manfredi E. Portación y caracterización de Staphylococcus aureus en manipuladores de alimentos. Rev. argent. microbiol. 2012; 44: 101-104.

(20) Ultrich RG. Evolving superantigens Staphylococcus aureus. FEMS Immunol. Med. Microbiol. 2000; 27: 1-7. DOI: http://doi. org/10.1111/j.1574-695X.2000.tb01404.x.

(21) Balaban N., Rasooly A. Staphylococcal enterotoxins. Int. J. Food Microbiol. 2000; 61: 1-10. DOI: http://doi.org/10.1016/S01681605(00)00377-9.

(22) Asao T., Kumeda Y., Kawai T., Shibata T., Oda H., Haruki K., et al. An extensive outbreak of staphylococcal food poisoning due to low-fat milk in Japan: estimation of enterotoxin $A$ in the incriminated milk and powdered skim milk. Epidemiol.
Infect. 2003; 130: 33-40. DOI: http://doi.org/10.1017/ S0950268802007951.

(23) Boerema JA., Clemens R., Brightwell G. Evaluation of molecular methods to determine enterotoxigenic status and molecular genotype of bovine, ovine, human and food isolates of Staphylococcus aureus. Int. J. Food Microbiol. 2006; 107(2): 192201. DOI: http://doi.org/10.1016/j.ijfoodmicro.2005.07.008.

(24) Manfredi EA., Leotta GA., Rivas M. PCR múltiple para la detección de los genes sea, seb, sec, sed y see de Staphylococcus aureus Caracterización de aislamientos de origen alimentario. Rev. argent. microbiol. 2010; 42: 212-215.

(25) Moore J., Heaney N., Millar B., Crowe M., Elborn J. Incidence of Pseudomonas aeruginosa in recreational and hydrotherapy pools. Commun Dis Public Health. 2002; 5: 23-26.

(26) Bello-Pérez LA., Ortiz-Dillanes DMA., Pérez-Memije E., CastroDomínguez V. Salmonella en carnes crudas: un estudio en localidades del Estado de Guerrero. Salud Pública de Méx. 1990; 32(1): 74-79.

(27) Blanco-Ríos F., Casadiego-Ardila G., Pacheco PA. Calidad microbiológica de alimentos remitidos a un laboratorio de salud pública en el año 2009. Rev. salud pública. 2011; 13(6): $953-$ 965. DOI: http://doi.org/10.1590/S0124-00642011000600008.

(28) Franco-Monsreal J., Lara-Zaragoza EB., Villa-Ruano N., MotaMagaña L., Del Socorro LE., Serralta-Peraza S., et al. Especies de importancia clínica del género Vibrio en alimentos marinos de origen animal de establecimientos de Puerto Ángel, Oaxaca, México. Ciencia y Mar. 2014; 20(52): 3-30.

(29) Fernández FS., Alonso G. Cólera y Vibrio cholerae. INHRR. 2009; 40(2): 50-69.

(30) Ávila-Quezada G., Sánchez E., Muñoz E., Martínez LR., Villalobos E. Diagnóstico de la calidad microbiológica de frutas y hortalizas en Chihuahua, México. Phyton (B. Aires). 2008; 77: 129-136.

(31) Salgado CMT. Manual de laboratorio de microbiología general. U. de Caldas, Manizales, Caldas. 2007.

(32) Velasco J., Araque M., Araujo E., Longa A., Nieves B., Ramírez AC., et al. Manual práctico de bacteriología clínica. Primera edición digital. Universidad de Los Andes, Mérida, Venezuela. 2011.

(33) González FS., Villagra TA., Pichel M., Figueroa S., Merletti G., Caffer MI., et al. Binsztein N. Caracterización de aislamientos de Vibrio cholerae no-O1, no-O139 asociados a cuadros de diarrea. Rev. Argent. Microbiol. 2009; 41(1): 11-17.

(34) Serrano AFJ. Manual Práctico de parasitología veterinaria. Universidad de Extremadura, Badajoz, Extremadura, España, 2010.

(35) Figueroa GG., Navarrete PW., Caro MC., Troncoso M., Faúndez ZG. Portación de Staphylococcus aureus enterotoxigénicos en manipuladores de alimentos. Rev. Med. Chile. 2002; 130: 859864. DOI: http://doi.org/10.4067/S0034-98872002000800003.

(36) Mejía NMR., Silvia MA., Suazo H., Catarino CJ., Rodríguez E., Espinal O., et al. Brote por Staphylococcus aureus en una guardería infantil en Choluteca, Honduras. Rev Med Hondur. 2009; 77(2): 57-98.

(37) Organización de las Naciones Unidas para la Agricultura y la Alimentación (FAO, 2009). Enfermedades transmitidas por

96 - Revista Ciencia y Agricultura (Rev. Cien. Agri.) Vol. 14 (2). ISSN 0122-8420. Julio - Diciembre 2017, pp. 89-97. Tunja (Boyacá) - Colombia. 
alimentos y su impacto socioeconómico. Estudios de caso en Costa Rica, El Salvador, Guatemala, Honduras y Nicaragua. Informe técnico sobre ingeniería agrícola y alimentaria, 2009. Roma.

(38) Zendejas-Manzo GS., Avalos-Flores H., Soto-Padilla MY. Microbiología general de Staphylococcus aureus: Generalidades, patogenicidad y métodos de identificación. Rev Biomed. 2014; 25(3): 129-143.

(39) Ayccek H., Cakiroglu S., Stevenson TH. Incidence of Staphylococcus aureus in ready-to-eat meals from military cafeterias in Ankara, Turkey. Food Control. 2005; 16(6): 531-534. DOI: http://doi. org/10.1016/j.foodcont.2004.04.005.

(40) Carreño JN., Gaona MA., Peña MC., Ríos DI., Rivera C. Infecciones recurrentes por Staphylococcus aureus en paciente con piercing nasal asociado al estado de portador: Estudio de caso. Rev. Cienc. Salud. 2006; 4(2): 116-122.

(41) Kim H., Griffiths M., Fazil A., Lammerding A. Probabilistic risk model for staphylococcal intoxication from pork-based food dishes prepared in food service Establishments in Korea. J. Food Prot. 2009, 72(9): 1897-908. DOI: http://doi.org/10.4315/0362028X-72.9.1897.

(42) Méndez IA., Badillo CA., Ortiz PG., Faccini AA. Caracterización microbiológica de Salmonella en alimentos de venta callejera en un sector universitario de Bogotá, Colombia. julio a octubre de 2010. Méd. UIS. 2011; 24(1): 23-29.

(43) Puerta-García A., Mateos-Rodríguez F. Enterobacterias. Medicine. 2010; 10(51): 3426-3431. DOI: http://doi.org/10.1016/S03045412(10)70056-1.

(44) Durango J., Arrieta G., Mattar S. Presencia de Salmonella spp. en un área del Caribe colombiano: un riesgo para la salud pública. Biomédica. 2004; 24: 89-96. DOI: http://doi.org/10.7705/ biomedica.v24i1.1252.

(45) Trejos-Suárez J., Castaño-Osorio JC. Factores de virulencia del patógeno intestinal Entamoeba histolytica. Infectio. 2009; 13(2): 100-110. DOI: http://doi.org/10.1016/S0123-9392(09)70731-3.

(46) Devera R., Blanco Y., González H., García L. Parásitos intestinales en lechugas comercializadas en mercados populares $y$ supermercados de Ciudad Bolívar, Estado Bolívar, Venezuela. Rev. Soc. Ven. Microbiol. 2006; 26(2): 396-408.

(47) Monge R., Chinchilla M., Reyes L. Estacionalidad de parásitos y bacterias intestinales en hortalizas que se consumen crudas en Costa Rica. Rev. Biol. Trop. 1996; 44(2): 369-375.

(48) Lozano SSL. Parasitosis de transmisión directa en personal manipulador de alimentos bajo un programa de salud ocupacional en el Distrito de Santa Marta durante el año 2006. Revista de la Facultad de Ciencias de la Salud. 2009; 6(2): 112117.

(49) Bayona MA. Prevalencia de salmonella y enteroparásitos en alimentos y manipuladores de alimentos de ventas ambulantes y restaurantes en un sector del norte de Bogotá, Colombia. Rev. U.D.C.A Act. \& Div. Cient. 2012; 15(2): 267-274.

(50) Arévalo Z., Clavijo AM., Rolo de M., Álvarez M., Conroy D., Infante D., et al. Aislamiento de Vibrio cholerae a partir de lisas y tilapias en Venezuela. Rev. Soc. Ven. Microbiol. 2003; 23(2): 127-130.
(51) Baron S., Lesne J., Jouy E., Larvor E., Kempf I., Boncy J., et al. Antimicrobial susceptibility of autochthonous aquatic Vibrio cholerae in Haiti. Front Microbiol. 2016; 7: 1671. DOI: http:// doi.org/10.3389/fmicb.2016.01671.

(52) Fernández-Delgado M., Rojas H., Duque Z., Suárez P., Contreras M., García-Amado MA., et al. Biofilm formation of Vibrio cholerae on stainless steel used in food processing. Rev. Inst. Med. Trop. 2016; 58-47.

(53) Rizzo L., Manaia C., Merlin C., Schwartz T., Dagot C., Ploy MC., et al. Urban wastewater treatment plants as hotspots for antibiotic resistant bacteria and genes spread into the environment: a review. Sci. Tot. Environ. 2013; 447, 345-360. DOI: http://doi. org/10.1016/j.scitotenv.2013.01.032.

(54) Schoolnik GK., Yildiz FH. The complete genome sequence of Vibrio cholerae: a tale of two chromosomes and of two lifestyles. Genome Biology. 2000. 1(3): reviews1016.1-reviews1016.3. DOI: http://doi.org/10.1186/gb-2000-1-3-reviews1016.

(55) Flórez AC., Rincón C., Garzón P., Vargas N., Enríquez C. Factores relacionados con enfermedades transmitidas por alimentos en restaurantes de cinco ciudades de Colombia, 2007. Infectio. 2008; 12(4): 255-266. 